\title{
The finger lesion
}

\author{
Pouya Entezari $^{1} \cdot$ Ameneh Alaini $^{2} \cdot$ Yahya Daneshbod $^{3} \cdot$ Hadi Mirfazaelian $^{1}$
}

Received: 27 May 2015/Accepted: 6 August 2015/Published online: 28 September 2015

(C) SIMI 2015

A 35-year-old farmer presented with a skin lesion on his left hand. He reported that the lesion appeared about 8 months prior. It was followed by other growing lesions along with no improvement with antibiotic treatment. On physical examination, there was a $1.7 \times 1 \mathrm{~cm}$ lesion on dorsum of the long finger on the left hand (Fig. 1). The lesion was nodular, erythematous, firm with small ulcers. There was no lymphadenopathy or fever. Because of the persistence of the lesion and its antibiotic resistance, a biopsy was performed. Histopathological examination revealed an inflammatory process with a granulomatous reaction without any evidence for malignancy or a specific microorganism. On skin tissue culture, fungal colonies grew that were identified as Sporothrix schenckii.

Sporotrichosis is a chronic subcutaneous infection caused by the fungal agent Sporothrix schenckii [1]. The pathogen is acquired mostly by traumatic inoculation with soil, plants, or other organic materials mainly in gardeners or agriculturists [2]. The most common clinical presentation of disease is lymphocutaneous, which is seen in $70-80 \%$ of cases [3]. Besides skin, sporotrichosis can involve any other tissues such as lymphatic vessels, bones (predominantly in immunocompromised patients), cartilage and muscle [4]. It is noteworthy that disseminated and systemic forms of the disease are mostly associated with immunodeficiency [5]. Skin lesions in sporotrichosis are

Hadi Mirfazaelian

Hadimir_43@yahoo.com

1 Department of Emergency Medicine, Tehran University of Medical Sciences, Tehran, Iran

2 Department of Dermatology, Arak University of Medical Sciences, Arak, Iran

3 Dr. Daneshbod Pathology Laboratory, Shiraz, Iran

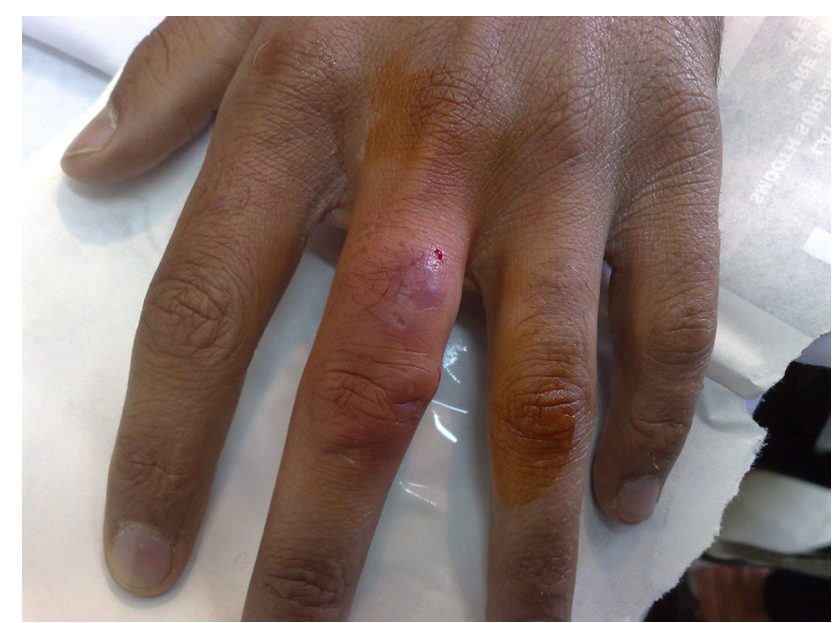

Fig. 1 The skin examination showed a $1.7 \times 1 \mathrm{~cm}$ erythematosus, firm nodule with small ulcers on dorsal of the left third finger

highly variable including erythema, nodules or ulcers at the point of inoculation and also along lymphatic vessels $[1,6]$. Regarding various clinical manifestations of the disease, it is often difficult to diagnose it only by visual inspection of lesions and imaging appearances are unable to assist in the identification of the type of microorganism. Thus, mycological culture and clinical history are required in any clinical presentation, to confirm the diagnosis [6]. Incision and drainage is not the definitive treatment, but more of an adjunct. Oral itraconazole for 3-6 months is the most common and useful treatment for sporotrichosis [7]. In case of immunodeficiency or disseminated infection, administration of systemic amphotericin B is required [8].

The patient was administered with Itraconazole for 3 months. The patient refused to attend the clinic for follow-up visits. 


\section{Compliance with ethical standards}

Conflict of interest Authors declare that they have no conflict of interest to the article.

Statement of human and animal rights All procedures performed in studies involving human participants were in accordance with the ethical standards of the institutional and/or national research committee and with the 1964 Helsinki declaration and its later amendments or comparable ethical standards. This article does not contain any studies with animals performed by any of the authors.

Informed consent Informed consent was obtained from the involved patient in case report.

\section{References}

1. Yamaguchi T, Ito S, Takano Y, Umeda N, Goto M, Horikoshi M et al (2012) A case of disseminated sporotrichosis treated with prednisolone, immunosuppressants, and tocilizumab under the diagnosis of rheumatoid arthritis. Intern Med (Tokyo, Japan) 51(15):2035-2039
2. Barros MB, de Almeida Paes R, Schubach AO (2011) Sporothrix schenckii and sporotrichosis. Clin Microbiol Rev 24(4):633-654

3. Rafal ES, Rasmussen JE (1991) An unusual presentation of fixed cutaneous sporotrichosis: a case report and review of the literature. J Am Acad Dermatol 25(5 Pt 2):928-932

4. Queiroz-Telles F, Nucci M, Colombo AL, Tobon A, Restrepo A (2011) Mycoses of implantation in Latin America: an overview of epidemiology, clinical manifestations, diagnosis and treatment. Med Mycol 49(3):225-236

5. Chang S, Hersh AM, Naughton G, Mullins K, Fung MA, Sharon VR (2013) Disseminated cutaneous sporotrichosis. Dermatol Online J 19(11):20401

6. Kauffman CA, Bustamante B, Chapman SW, Pappas PG (2007) Clinical practice guidelines for the management of sporotrichosis: 2007 update by the Infectious Diseases Society of America. Clin Infect Dis 45(10):1255-1265

7. de Lima Barros MB, Schubach AO, de Vasconcellos Carvalhaes de Oliveira R, Martins EB, Teixeira JL, Wanke B (2011) Treatment of cutaneous sporotrichosis with itraconazole-study of 645 patients. Clin Infect Dis 52(12):e200-e206

8. Goldani LZ, Aquino VR, Dargel AA (1999) Disseminated cutaneous sporotrichosis in an AIDS patient receiving maintenance therapy with fluconazole for previous cryptococcal meningitis. Clin Infect Dis 28(6):1337-1338 